\title{
Structural Insights into the Inhibition of Amyloid Fibril Formation by Fibrinogen via Interaction with Prefibrillar Intermediates
}

Naoki Yamamoto $^{\dagger \S}$, Taiki Akai ${ }^{\dagger}$, Rintaro Inoue ${ }^{\ddagger}$, Masaaki Sugiyama ${ }^{\ddagger}$, Atsuo Tamura ${ }^{\dagger}$, and Eri Chatani $^{\dagger}$

${ }^{\dagger}$ Graduate School of Science, Kobe University, 1-1 Rokkodai-cho, Nada-ku, Kobe 657-8501, Japan

${ }^{\ddagger}$ Institute for Integrated Radiation and Nuclear Science, Kyoto University, 2, Asashiro-Nishi, Kumatori-cho, Sennan-gun, Osaka 590-0494, Japan

Figure S1. Negligible interactions of Fg with insulin B chain in a form of monomers or amyloid fibrils.

Figure S2. Inhibition of fibril formation of B chain by the addition of Fg after the preformation of prefibrillar intermediates.

Figure S3. Time dependence of the hydrodynamic diameter in the formation of the B-chain prefibrillar intermediates of B chain with or without Fg.

Figure S4. Prevention of dissociation of the B chain prefibrillar intermediates complexed with Fg upon dilution.

Figure S5. Hydrodynamic diameter distributions of the prefibrillar intermediates of B chain complexed with Fg.

Figure S6. SAXS analysis of Fg-B chain complex that was formed by a simultaneous incubation of Fg and B chain. 

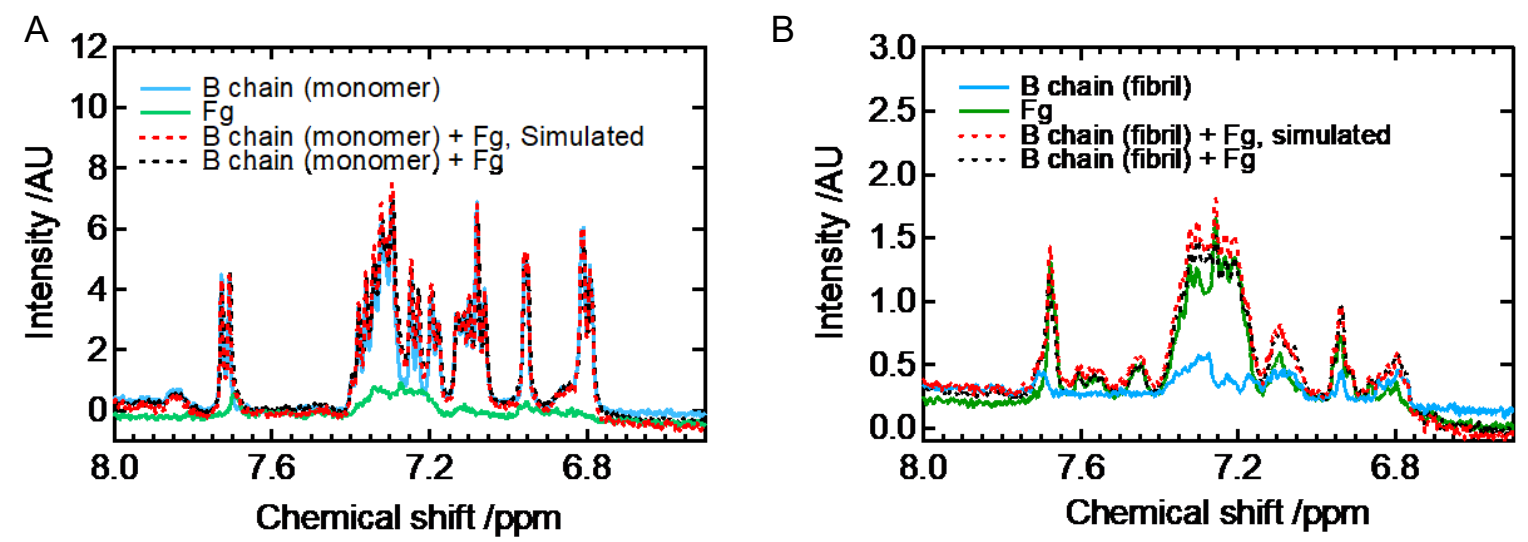

Figure S1. Negligible interactions of Fg with insulin B chain in a form of monomers (A) or amyloid fibrils (B).

(A) NMR spectrum of monomeric B chain incubated with fibrinogen (Fg) (black line). In this measurement, the concentration of B chain was $0.35 \mathrm{mg} / \mathrm{ml}$, where B chain was kept monomeric. ${ }^{1}$ The final concentration of Fg was $0.875 \mathrm{mg} / \mathrm{ml}$. The spectrum is nearly the same as the simulated spectrum where the individual spectra of B chain and Fg were summed (red line), suggesting no interactions between Fg and monomeric B chain. (B) The NMR spectrum of Fg incubated with B-chain amyloid fibrils (black line). The final concentration of Fg and the amyloid fibrils were $0.75 \mathrm{mg} / \mathrm{ml}$ and $1.75 \mathrm{mg} / \mathrm{ml}$, respectively. Red line shows the simulated spectrum where these spectra were summed, which is almost identical to the black line suggesting no interactions between Fg and B chain amyloid fibrils. 
A

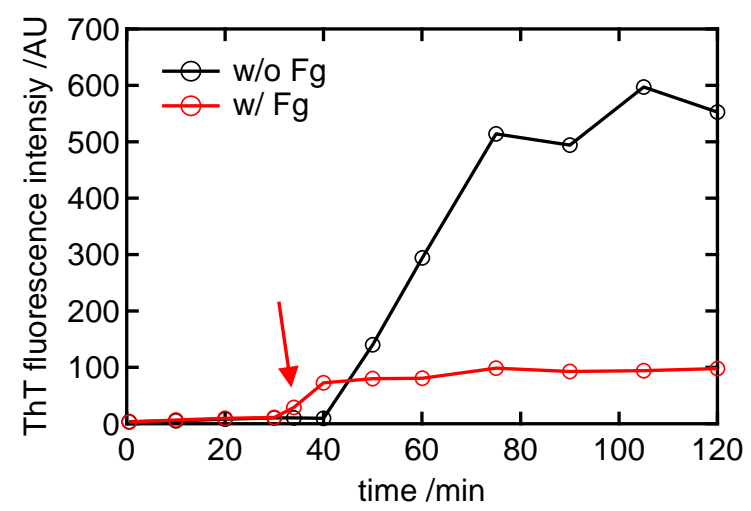

B

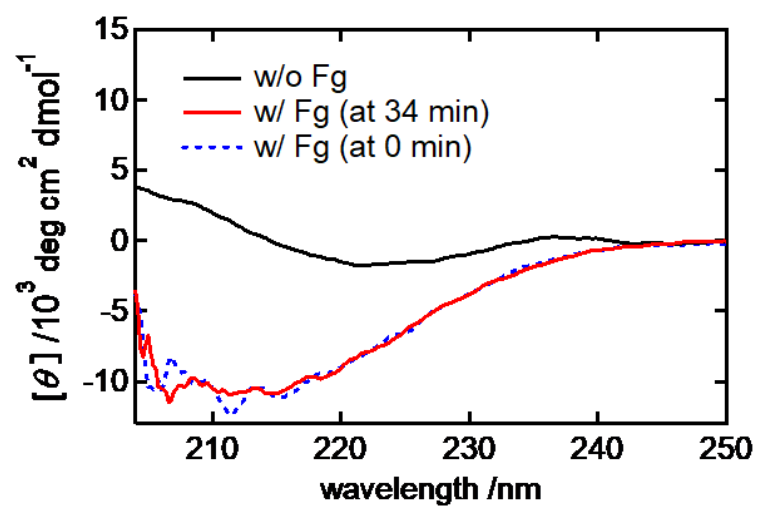

Figure S2. Inhibition of fibril formation of B chain by the addition of Fg after the preformation of prefibrillar intermediates.

(A) The time course of B chain fibrillation monitored by ThT fluorescence intensity. At a time point of $34 \mathrm{~min}$ (as indicated by an arrow), Fg or buffer for a control was added to the reaction sample. When buffer was added, marked increase in ThT fluorescence intensity was observed (black line), indicating fibril formation. On the other hand, when Fg was added, ThT fluorescence intensity kept constant, although jumped slightly to a higher value immediately after the Fg addition (red line). This result suggests that Fg inhibits fibril formation even after the formation of prefibrillar intermediates. (B) CD spectra of samples in which Fg or buffer was added after the 34-min preincubation (red and black lines, respectively). The samples were taken at $90 \mathrm{~min}$, and the spectrum contribution of Fg was subtracted for the sample in the presence of Fg. The CD spectrum of the sample incubated with Fg from the beginning (blue line) was also shown to compare the spectral shape. The spectral similarity between the red and the blue lines verifies the suggestion that the Fg inhibits fibril formation even after the prefibrillar intermediates are formed. 
A

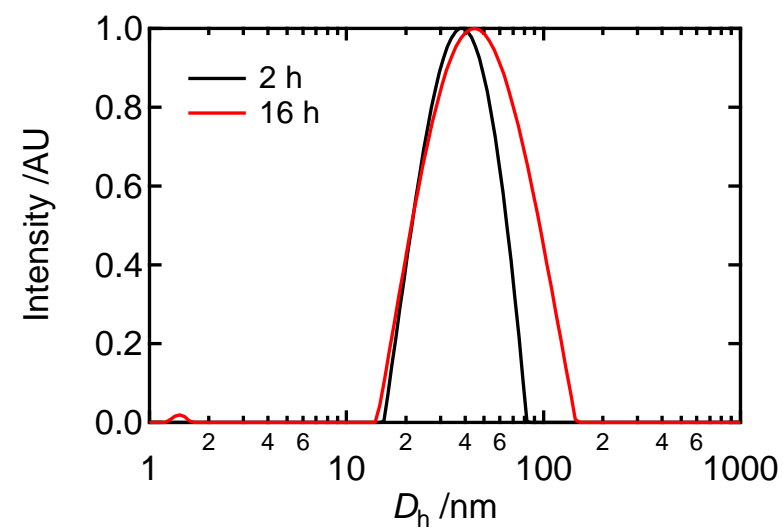

B

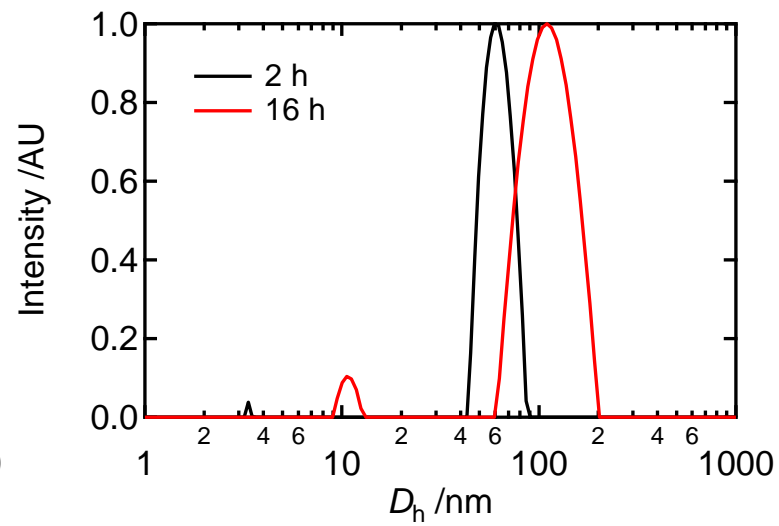

Figure S3. Time dependence of the hydrodynamic diameter in the formation of the Bchain prefibrillar intermediates of $B$ chain with (A) or without $\mathrm{Fg}(\mathrm{B})$.

The reaction of the prefibrillar intermediate formation of $\mathrm{B}$ chain was performed by incubating $1.4 \mathrm{mg} / \mathrm{ml} \mathrm{B}$ chain under quiescent conditions with or without $3.5 \mathrm{mg} / \mathrm{ml} \mathrm{Fg}$. The samples were taken at $2 \mathrm{~h}$ and $16 \mathrm{~h}$, and distributions of the hydrodynamic diameter $D_{\mathrm{h}}$ were analyzed by DLS. It is evident that the size development of B-chain prefibrillar intermediates incubated with Fg became much slower compared to the situation where Fg was not co-incubated. 

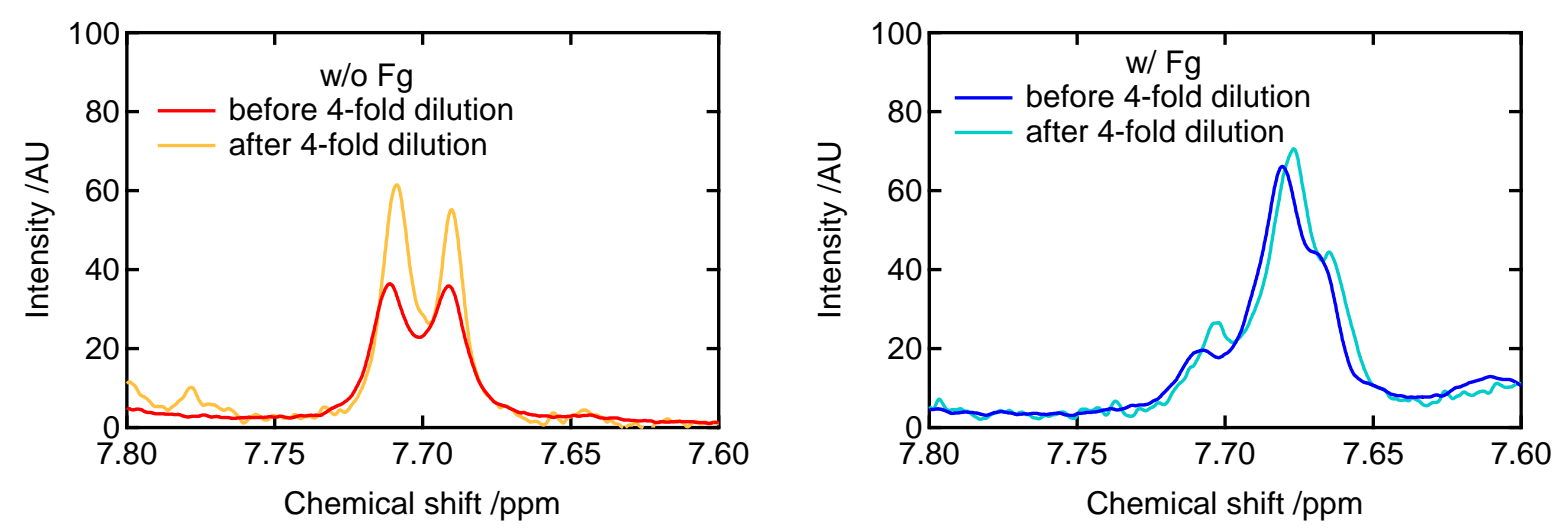

\section{Figure S4. Prevention of dissociation of the $B$ chain prefibrillar intermediates complexed with Fg upon dilution.}

(A) NMR spectra of histidine $\varepsilon$ protons of the prefibrillar intermediates of B chain before and after 4-fold dilution. B chain at the concentration of $1.4 \mathrm{mg} / \mathrm{ml}$ was incubated for $2.5 \mathrm{~h}$ at $25{ }^{\circ} \mathrm{C}$ under quiescent conditions to form the prefibrillar intermediates, which were then diluted by 4-fold with a same buffer. (B) NMR spectra of the prefibrillar intermediates of B chain complexed with Fg before and after 4-fold dilution. The chemical-shift range is same as that of panel A. B chain at the concentration of $1.4 \mathrm{mg} / \mathrm{ml}$ was incubated with $10 \mathrm{mg} / \mathrm{ml}$ $\mathrm{Fg}$ for $2.5 \mathrm{~h}$ at $25^{\circ} \mathrm{C}$ under quiescent conditions, and then the sample was diluted by 4 -fold with a same buffer. In both panels, the spectral intensity after the dilution was multiplied by 4 in order to normalize by protein concentration. 


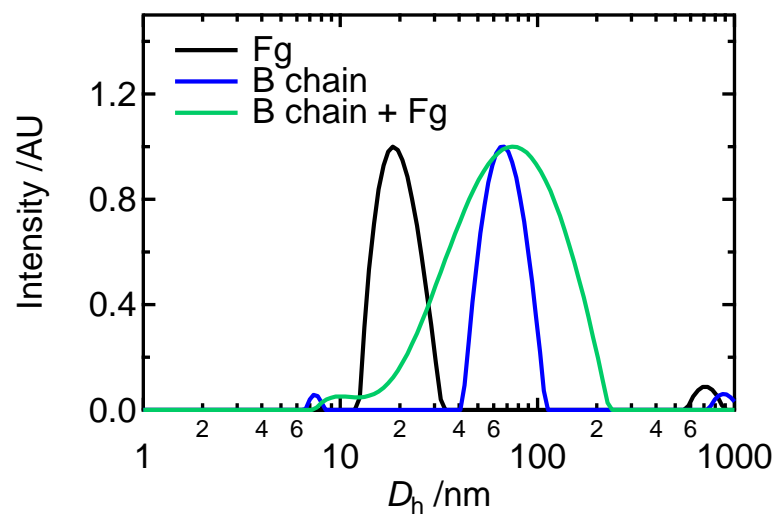

Figure S5. Hydrodynamic diameter distributions of the prefibrillar intermediates of B chain complexed with Fg.

The distributions of hydrodynamic diameters of the Fg, B chain, and B chain-Fg complex are shown. The same samples with those analyzed by SAXS were used for these DLS measurements. In this Figure, one of the repeated results is shown for each sample. The average hydrodynamic diameters are shown in Table 1 in the main text. 
A

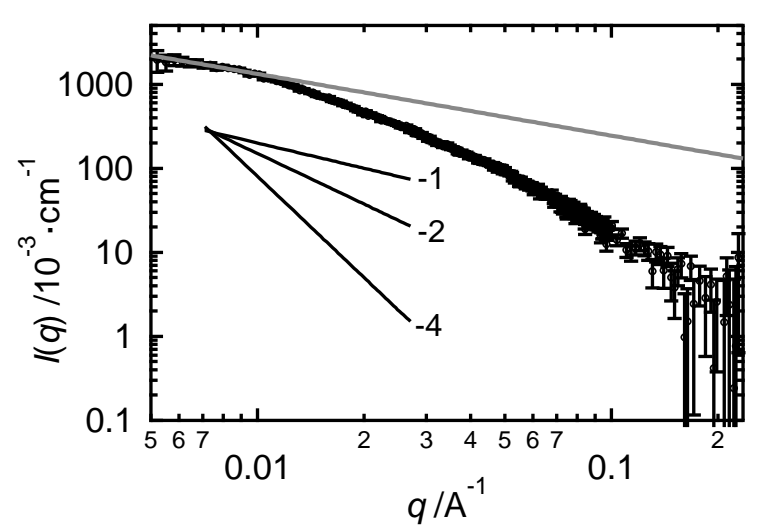

B

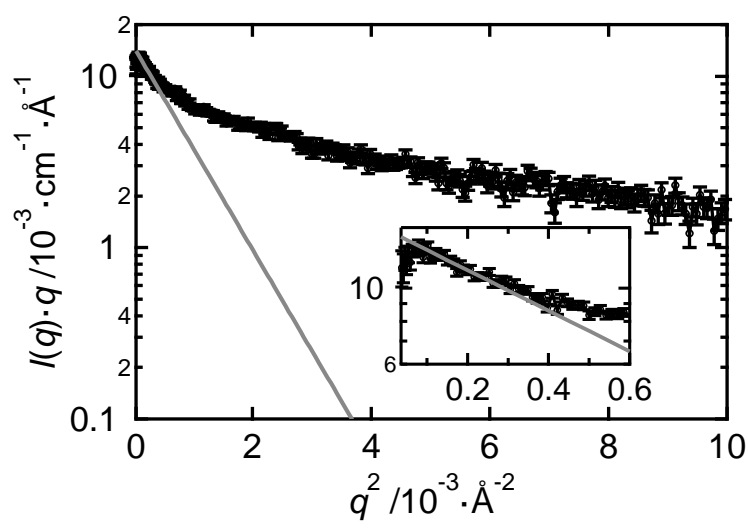

Figure S6. SAXS analysis of Fg-B chain complex that was formed by a simultaneous incubation of Fg and $B$ chain.

Fg and B chain were mixed at the concentration of $3.5 \mathrm{mg} / \mathrm{ml}$ and $1.4 \mathrm{mg} / \mathrm{ml}$, respectively. 2 hours after the incubation, the SAXS experiment was performed. (A) The log-log plot of $\mathrm{I}(q)$ vs $q$. Simulated slopes of $-1,-2$, or -4 were drawn in the Figure. (B) The cross-section plot to obtain $R_{\mathrm{c}}$. The inset shows the magnified low- $q$ region. The resultant value of $R_{\mathrm{c}}$ was $52.2 \pm 2.3 \AA$, by which the value of $R_{\mathrm{p}}$ became $73.8 \pm 3.3 \AA$. The hydrodynamic diameter $D_{\mathrm{h}}$ was $350 \pm 10 \AA$ based on a DLS measurement. As a result, the length of the complex was calculated to be $870 \pm 50 \AA$. 


\section{REFERENCE}

1. Yamamoto, N, Tsuhara, S, Tamura, A, and Chatani, E. (2018). A specific form of prefibrillar aggregates that functions as a precursor of amyloid nucleation. Sci. Rep. 8, 62 . 